\title{
Single nucleotide polymorphism in the $R A D 18$ gene and risk of colorectal cancer in the Japanese population
}

\author{
HIROTAKA KANZAKI ${ }^{1}$, MAMORU OUCHIDA $^{1}$, HIROKO HANAFUSA ${ }^{1}$, AKIKO SAKAI $^{1}$, \\ HIROMASA YAMAMOTO ${ }^{2}$, HIROMITSU SUZUKI ${ }^{2}$, MASAAKI YANO ${ }^{2}$, MOTOI AOE ${ }^{2}$, \\ KAZUE IMAI $^{3}$, HIROSHI DATE ${ }^{2}$, KEI NAKACHI $^{3}$ and KENJI SHIMIZU ${ }^{1}$
}

\begin{abstract}
${ }^{1}$ Department of Molecular Genetics and ${ }^{2}$ Department of Cancer and Thoracic Surgery, Graduate School of Medicine, Dentistry and Pharmaceutical Sciences, Okayama University, Okayama 700-8558; ${ }^{3}$ Department of Radiobiology/ Molecular Epidemiology, Radiation Effects Research Foundation, Hiroshima 732-0815, Japan
\end{abstract}

Received June 5, 2007; Accepted July 30, 2007

\begin{abstract}
The RAD18 gene, located on the human chromosome 3p24-p25, plays a crucial role in post-replication repair (PRR) in various organisms from yeast to humans. In the human $R A D 18$ gene, one coding single nucleotide polymorphism (SNP) at codon 302, encoding either arginine (Arg, CGA) or glutamine (Gln, CAA), was reported. Although the molecular function of the RAD18 protein came to be elucidated, the association between the RAD18 Arg302Gln polymorphism and the risk of human cancer development was not examined. Therefore, we investigated the relationship between the polymorphism and the development of human primary colorectal cancer (CRC). The Arg302Gln polymorphism in 100 patients with CRC and 200 healthy controls were genotyped by the polymerase chain reaction with confronting two-pair primer (PCR-CTPP) assay. The Gln/Gln genotype was significantly more frequent in CRC $(18.0 \%)$ than in the healthy controls $(11.5 \%)(\mathrm{p}=0.046)$. The increased risk was detected in CRC patients with the Gln/Gln genotype (Odds ratio [OR], 2.10; 95\% confidence interval [CI], 1.00 to 4.40). When the relationship of the SNP with clinicopathological parameters of CRC was investigated, particularly in the well-differentiated grade and in the lymph node metastasis (N1) CRC patients, significantly higher risks were detected (OR, 7.00; 95\% CI, 1.19-41.1 and OR, 3.71; 95\% CI, 1.3010.6, respectively). These results suggested that the RAD18 Arg302Gln polymorphism is associated with the risk of CRC. This report provides evidence for an association
\end{abstract}

Correspondence to: Dr Mamoru Ouchida, Department of Molecular Genetics, Graduate School of Medicine, Dentistry and Pharmaceutical Sciences, Okayama University, 2-5-1 Shikata-cho, Okayama 700-8558, Japan

E-mail: ouchidam@md.okayama-u.ac.jp

Key words: single nucleotide polymorphism, RAD18, postreplication repair, colorectal cancer, cancer predisposition between the RAD18 Arg302Gln polymorphism and human CRC risk.

\section{Introduction}

Any DNA damage induced by mutagens, such as UV light and mutagenic chemicals, must be repaired by DNA repair systems (1). However, when the DNA repair systems are stalled or saturated and such DNA damage is therefore not removed before the onset of DNA replication, single-stranded gaps are generated. These gaps will be filled by the post-replication repair (PRR) system. The molecular mechanisms of PRR are less clearly elucidated in comparison to the other repair pathways. The RAD6 pathway is known to be central to PRR (2). The genes belonging to the $R A D 6$ epistasis group, such as the RAD5, RAD18, RAD30, MMS2 and UBC13 genes, are all involved in the pathway. Among these gene products, RAD6 and RAD18 are two of the most important proteins and play a central role in this pathway. RAD6 is a ubiquitin-conjugating enzyme (E2) in the proteasome protein degradation system (3-5). RAD6 forms a tight complex with RAD18 (6-8), which is a single-strand DNA binding protein with a RINGfinger domain (9) and ubiquitin-ligating enzymes (E3). Although RAD6 interacts with several E3, the interaction with RAD18 is essential for carrying out PRR $(5,6,10,11)$. Since RAD6 interacts tightly with RAD18, although RAD6 does not have any DNA binding activity, it is proposed that RAD18 recruits RAD6 to the site of DNA damage via its physical interaction, where RAD6 and its complex then modulate stalled DNA replication through their ubiquitinconjugating activity. There are reports that the proliferating cell nuclear antigen (PCNA), a DNA polymerase sliding clamp that is involved in DNA synthesis and repair, is a substrate of the ubiquitin-conjugating enzyme and it is ubiquitinated in a RAD6- and RAD18-dependent manner. Therefore, the monoubiquitination of PCNA through RAD6 and RAD18 is necessary for carrying out DNA PRR (1216).

It was also reported that RAD18 knockout cells of mouse embryonic stem cells (17) and of chicken DT40 cells (18) were hypersensitive to various DNA-damaging agents and showed 
defective PRR. These cells also showed genomic instability demonstrated by increased rates of the sister chromatid exchange and integration of the exogenous DNA $(17,18)$. Therefore, the dysfunction of RAD18 increases the frequency of homologous recombination and illegitimate recombination and RAD18 contributes to the maintenance of genomic stability through PRR (19). The dysfunction of RAD18 is also thought to lead to the development of cancer $(1,20)$.

The genetic polymorphisms of DNA repair genes were analyzed to determine the susceptibility to several cancers, including lung $(21,22)$, colorectal $(23)$, head and neck (24), breast (25), bladder cancer (26) and leukemia (27). The RAD18 gene is known to have a single nucleotide polymorphism (SNP) at codon 302, encoding either arginine (CGA) or glutamine (CAA), while the function of this SNP and its molecular mechanism is not clarified. We conducted a pilot study to see whether the RAD18 Arg302Gln polymorphism is associated with cancer-incidence and found a significant correlation with CRC. This report provides evidence for an association between the RAD18 Arg302Gln polymorphism and human CRC risk.

\section{Materials and methods}

Subjects. We analyzed 100 Japanese patients chosen from those who were histologically diagnosed as having primary colorectal cancer and who underwent surgical operation at Okayama University Hospital (Okayama, Japan) in 1994-2003. Patients were gathered not only from Okayama but also from 10 other prefectures in Western Japan, mainly the Chugoku and Shikoku Districts (around Okayama). We confirmed that all CRC patients have primary colorectal carcinomas by microscope. Clinical stage and pathological grade in all CRC patients were confirmed by operation and pathology. The clinicopathological staging and histological classification were according to the criteria of the UICC Tumor-Node-Metastasis Classification of Malignant Tumor (TNM), $6^{\text {th }}$ edition, 2002, colon and rectum (ICD-O C18-C20). For the controls, each of the 200 healthy controls we analyzed were selected by computer-aided randomization, all of which were from the subjects of cohort studies on a Japanese general population $>40$ years of age in a town near the Saitama Cancer Center. The residents in this town are neither genetically nor demographically close, i.e., its population increased because of a population influx from other areas, with a social increase rate of about $5 \%$ every year for 15 years. Written informed consent was obtained from all of the cancer patients and controls concerned. This study was approved by The Bioethics Committee of Okayama University Medical School. The characteristics of the 100 CRC patients and the 200 controls are shown in Table I. There were no significant differences in gender and age at recruitment between the CRC patients and the controls $(\mathrm{p} \geq 0.05)$. Pack-year equivalents [(cigarettes/ day/20) x (smoking years)] were used for smoking status (we could not obtain smoking status for 3 out of the 100 patients).

DNA extraction. The genomic DNA of cancer patients was isolated from the non-cancerous region of the resected specimens or from the mononuclear cells of the peripheral blood by the standard method of proteinase $\mathrm{K}$ digestion and
Table I. Characteristics of CRC patients and healthy controls.

\begin{tabular}{lccc}
\hline & $\begin{array}{c}\text { Patients } \\
\mathrm{n}(\%)(\mathrm{n}=100)\end{array}$ & $\begin{array}{c}\text { Controls } \\
\mathrm{n}(\%)(\mathrm{n}=200)\end{array}$ & $\mathrm{p}$-value \\
\hline Gender & & & 0.371 \\
$\quad$ Male & $61(61.0)$ & $133(66.5)$ & \\
Female & $39(39.0)$ & $67(33.5)$ & \\
Age (years $\pm \mathrm{SD})$ & $65.1 \pm 10.1$ & $65.6 \pm 9.42$ & \\
Smoking habit & & & $0.052^{\mathrm{a}}$ \\
No-smoker & $42(42.0)$ & $63(31.5)$ & \\
Smoker & $55(55.0)$ & $137(68.5)$ & \\
$\quad<20$ pack-years & $14(25.5)$ & $17(12.4)$ & \\
$\quad 220$ pack-years & $40(72.7)$ & $87(63.5)$ & \\
$\quad$ Unknown & $1(1.8)$ & $33(24.1)$ & \\
Unknown & $3(3.0)$ & $0(0.0)$ & \\
\hline
\end{tabular}

The mean age of each group with a standard deviation is shown. ${ }^{a}$ p-values are for the differences in the number of smokers and non-smokers between the patients and the controls.

phenolchloroform extraction. The genomic DNA of the healthy controls was extracted from the peripheral lymphocytes.

Genetic analysis. The RAD18 Arg302Gln polymorphism was detected by polymerase chain reaction using the confronting two-pair primer (PCR-CTPP) method $(28,29)$. According to the published sequence of the human $R A D 18$ gene, we designed two sets of paired primers. The first set of primers was as follows: forward primer 1, 5'-ATA CCC ATC ACC CAT CTT C-3' and reverse primer 1, 5'-GTC TTC TCT ATA TTT TCG ATT TCT T-3' for the Gln allele amplifying a 146-bp band. The second set of primers was as follows: forward primer 2, 5'-TTA ACA GCT GCT GAA ATA GTT CG-3' and reverse primer 2, 5'-CTG AAA TAG CCC ATT AAC ATA CA-3' for the Arg allele amplifying a 106-bp band. A 206-bp band was designed between the forward primer 1 and the reverse primer 2 . The reaction mixture $(20 \mu 1)$ contained $40 \mu \mathrm{M}$ of each dNTP, 1X PCR buffer, 8 pmol of the forward primer 1 and reverse primer 2, 24 pmol of the forward primer 2 and reverse primer 1, 20 ng of the genomic DNA and 0.5 units of the Taq DNA polymerase (Takara, Kyoto, Japan). The PCR amplification was initiated by a denaturing step at $94^{\circ} \mathrm{C}$ for $3 \mathrm{~min}$, followed by 35 cycles at $94^{\circ} \mathrm{C}$ for $30 \mathrm{sec}$, $64^{\circ} \mathrm{C}$ for $1 \mathrm{~min}, 72^{\circ} \mathrm{C}$ for $1 \mathrm{~min}$ and a final extension step at $72^{\circ} \mathrm{C}$ for $7 \mathrm{~min}$. For genotyping, the PCR products were subjected to electrophoresis in $3 \%$ agarose gel, stained with ethidium bromide and then visualized on a UV transilluminator. In order to confirm the allele types, certain PCR products were processed with the Big Dye terminator Cycle Sequencing Kit (Applied Biosystems, Foster City, CA, USA), then analyzed and confirmed on an ABI 3100 sequencer (Applied Biosystems).

Statistical analysis. We compared the allele frequencies of the Arg/Gln (CGA/CAA) polymorphism in the RAD18 gene between the healthy control group and the patient groups with 
CRC. The distribution of the RAD18 genotype (Arg/Arg, Arg/Gln, Gln/Gln) in all of the patients and the controls was tested for adherence to the Hardy-Weinberg equilibrium. The statistical analyses were conducted by the SPSS software Ver.12.0 (SPSS Inc., Tokyo, Japan). The Chi-square test was used to compare the genotype distribution between the cancer patients and the healthy controls. The odds ratio (OR) and 95\% confidence interval $(95 \% \mathrm{CI})$ were both adjusted for age, gender and smoking status using an unconditional logistic regression model. The relationship between the genotype and the clinicopathological parameters was examined by the Chisquare test and Fisher's exact probability test. A p-value of $<0.05$ was considered to be statistically significant.

\section{Results}

Assessment of cancer risk by RAD 18 genotyping. The characteristics of the 100 CRC patients and the 200 healthy controls are shown in Table I. There were no significant differences in gender, age or smoking status between the two groups. Pack-year equivalents were used for smoking status (however, we could not obtain the smoking status for 3 out of the 100 CRC patients).

Fig. 1A shows a representative polymerase chain reaction with confronting two pair primer (PCR-CTPP) assay patterns of the Arg/Gln (CGA/CAA) genotypes in the codon 302 of the RAD18 gene. The allele types were determined as follows: two fragments of 205- and 106-bp for the $\mathrm{Arg} / \mathrm{Arg}(\mathrm{G} / \mathrm{G})$ genotype, two fragments of 205- and 146-bp for the Gln/Gln (A/A) genotype and three fragments of 205-, 146- and 106-bp for the Arg/Gln (G/A) genotype. We confirmed that each PCR mixture contained no non-specific bands by electrophoresis in $3 \%$ agarose gel. The genotypes were confirmed by the subsequent sequencing of representative cases (Fig. 1B).

The genotype analysis of this SNP revealed that the genotype frequency was significantly different between the control and patient groups. The frequencies of the three genotypes in the RAD18 gene are shown in Table II. The frequencies of the genotypes Arg/Arg, Arg/Gln and Gln/Gln found were 43.0, 45.5 and $11.5 \%$ in the controls and 32.0, 50.0 and $18.0 \%$ in the CRC patients. All of the results fitted the Hardy-Weinberg equilibrium. Compared to the controls with
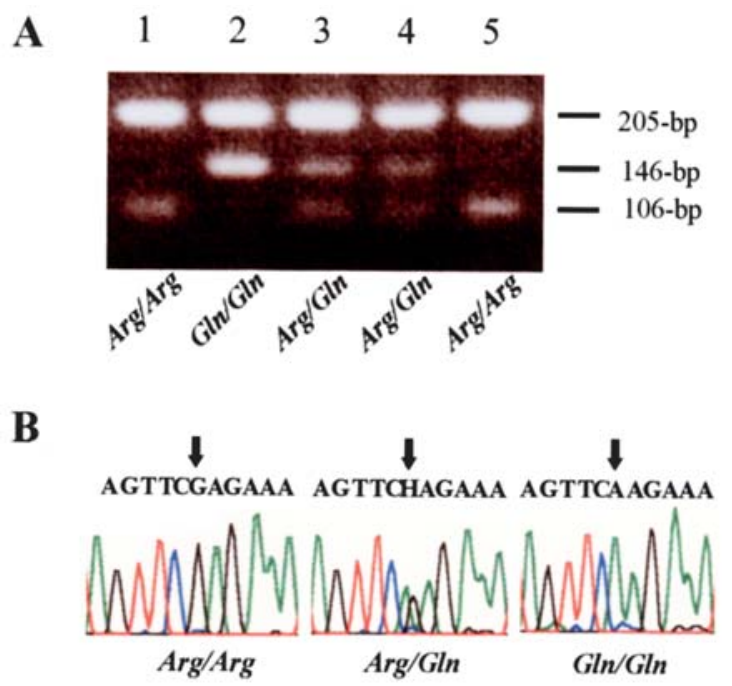

Figure 1. The single nucleotide polymorphism of the RAD18 gene at codon 302. (A) The PCR-CTPP patterns of the RAD18 SNP. The PCR product was electrophoresed in 3\% agarose gel. Two fragments of 205- and 106-bp show the $\operatorname{Arg} / \operatorname{Arg}(\mathrm{G} / \mathrm{G})$ genotype, two fragments of 205- and 146-bp show the $\mathrm{Gln} / \mathrm{G} \ln (\mathrm{A} / \mathrm{A})$ genotype and three fragments of 205-, 146- and 106-bp show the $\mathrm{Arg} / \mathrm{Gln}$ (G/A) genotype. The case number and genotypes are shown at the top and bottom, respectively. (B) DNA sequence patterns of the RAD18 SNP. Heterozygous signals (G/A) are shown by arrows in the Arg/Gln genotype.

the Arg/Arg genotype, CRC patients with the homozygous Gln/Gln genotype exhibited the most significantly increased risk with an odds ratio (OR) of 2.10 (95\% CI, 1.00-4.40), therefore suggesting that the homozygous Gln/Gln (A/A) genotype has an enhanced risk of CRC development.

The association between the RAD18 genotype and clinicopathological parameters in CRC patients. When we analyzed the relationship between the genotype distribution and the clinicopathological parameters of the CRC patient group, the well-differentiated grade and lymph node metastasis (N1) patients with the A/A genotype exhibited a significantly increased risk with an adjusted OR of 7.00 (95\% CI=1.1941.1) and 3.71 (95\%CI=1.30-10.6), respectively (Table III). There were no overall differences in genotype distribution within each parameter using the Chi-square test or the

Table II. The RAD18 genotypes in CRC patients and healthy controls.

\begin{tabular}{|c|c|c|c|c|}
\hline$R A D 18$ & Patients & Controls & p-value & OR $(95 \% \mathrm{CI})$ \\
\hline genotype & $\mathrm{n}(\%)$ & $\mathrm{n}(\%)$ & & \\
\hline Arg/Arg & $32(32.0)$ & $86(43.0)$ & & Ref. 1 \\
\hline Arg/Gln & $50(50.0)$ & $91(45.5)$ & $0.151^{\mathrm{a}}$ & $1.47(0.87-2.52)$ \\
\hline Gln/Gln & $18(18.0)$ & $23(11.5)$ & $0.046^{\mathrm{a}}$ & $2.10(1.00-4.40)$ \\
\hline Total & 100 & 200 & & \\
\hline Allele frequencies & & & 0.037 & \\
\hline Arg & $114(57.0)$ & $263(67.8)$ & & \\
\hline Gln & $86(43.0)$ & $137(34.2)$ & & \\
\hline
\end{tabular}

${ }^{\mathrm{a}} \mathrm{p}$-values are shown against Arg/Arg genotype between patients and controls. 
Table III. Association between the RAD18 genotype and clinicopathological parameters of CRC patients.

\begin{tabular}{|c|c|c|c|c|c|c|c|}
\hline \multirow[b]{2}{*}{ Characteristics } & \multicolumn{3}{|c|}{ Genotype (\%) } & \multirow[b]{2}{*}{ Total } & \multirow[b]{2}{*}{ p-value ${ }^{a}$} & \multicolumn{2}{|c|}{$\mathrm{OR}^{\mathrm{b}}(95 \% \mathrm{CI})$} \\
\hline & Arg/Arg & Arg/Gln & $\mathrm{Gln} / \mathrm{G} \ln$ & & & Arg/Gln & $\mathrm{Gln} / \mathrm{Gln}$ \\
\hline Differentiated grade & & & & & 0.172 & & \\
\hline Well & $2(11.1)$ & $11(61.1)$ & $5(27.8)$ & 18 & & $4.71(1.00-22.2)$ & $7.00(1.19-41.1)$ \\
\hline Moderate & $25(33.8)$ & $36(48.6)$ & $13(17.6)$ & 74 & & $1.31(0.72-2.39)$ & $1.68(0.72-3.89)$ \\
\hline Poor & $2(66.7)$ & $1(33.3)$ & $0(0.0)$ & 3 & & $\mathrm{NE}$ & NE \\
\hline Others & $3(60.0)$ & $2(40.0)$ & $0(0.0)$ & 5 & & & \\
\hline $\mathrm{T}$ & & & & & 0.007 & & \\
\hline Tis, T1, T2 & $0(0.0)$ & $10(71.4)$ & $4(28.6)$ & 14 & & $\mathrm{NE}$ & $\mathrm{NE}$ \\
\hline $\mathrm{T} 3, \mathrm{~T} 4$ & $32(37.2)$ & $40(46.5)$ & $14(16.3)$ & 86 & & $1.14(0.65-2.00)$ & $1.32(0.58-3.00)$ \\
\hline $\mathrm{N}$ & & & & & 0.097 & & \\
\hline No & $13(28.9)$ & $24(53.3)$ & $8(17.8)$ & 45 & & $1.55(0.73-3.31)$ & $1.82(0.63-5.18)$ \\
\hline $\mathrm{N} 1$ & $9(25.0)$ & $18(50.0)$ & $9(25.0)$ & 36 & & $1.91(0.81-4.51)$ & $3.71(1.30-10.6)$ \\
\hline N2 & $9(56.3)$ & $7(43.7)$ & $0(0.0)$ & 16 & & $0.75(0.27-2.10)$ & NE \\
\hline Unknown & $1(33.3)$ & $1(33.3)$ & $1(33.3)$ & 3 & & & \\
\hline M & & & & & 0.622 & & \\
\hline M0 & $20(29.9)$ & $34(50.7)$ & $13(19.4)$ & 67 & & $1.59(0.84-3.01)$ & $2.22(0.93-5.33)$ \\
\hline M1 & $12(38.7)$ & $15(48.4)$ & $4(12.9)$ & 31 & & $1.18(0.52-2.70)$ & $1.19(0.35-4.10)$ \\
\hline Unknown & $0(0.0)$ & $1(50.0)$ & $1(50.0)$ & 2 & & & \\
\hline TNM stage & & & & & 0.929 & & \\
\hline 0.I.II & $12(30.8)$ & $20(51.3)$ & 7 (17.9) & 39 & & $1.47(0.66-3.26)$ & $1.66(0.55-5.02)$ \\
\hline III.IV & $20(34.5)$ & $28(48.3)$ & $10(17.2)$ & 58 & & $1.35(0.71-2.60)$ & $1.84(0.75-4.53)$ \\
\hline Unknown & $0(0.0)$ & $2(66.7)$ & $1(33.3)$ & 3 & & & \\
\hline
\end{tabular}

${ }^{\mathrm{a}} \mathrm{p}$-values were calculated by the Chi-square test or the Fisher's exact test. ${ }^{\mathrm{b}}$ ORs were adjusted for age, gender and smoking status. The Arg/Arg genotype was defined as the reference. T, primary tumor; N, lymph node metastasis; M, distant metastasis; NE, not estimated.

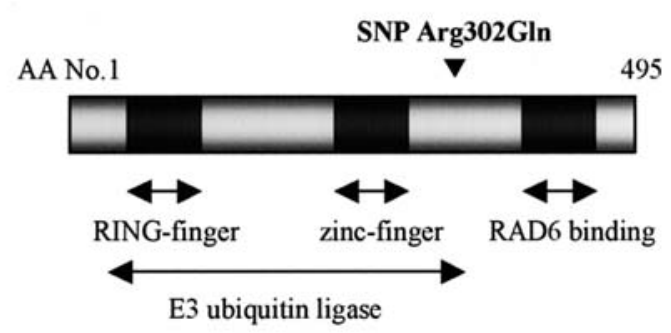

Figure 2. The location of the polymorphism and the functional motifs of RAD18. The SNP (Arg302Gln) is indicated by an arrowhead above the motif. The motifs of the RAD18 protein are depicted in dark gray and/or by arrows. The RING-finger, a really interesting new gene domain (AA25-63); zinc-finger, zinc-finger motif (AA201-223); RAD6 binding, RAD6 binding domain (AA371-410); E3 ubiquitin ligase, RING-finger-containing E3 ubiquitin-ligase domain (AA16-304). AA No., amino acid number.

Fisher's exact test, while the genotype distribution in the tumor stages may be biased due to the small sample size.

\section{Discussion}

In this study, we examined whether an SNP (Arg302Gln) in the $R A D 18$ gene is associated with the risk for the development, progression and metastasis of CRC. We found significant differences in the genotype distribution between the CRC patients and the healthy controls. Our findings suggest that the Gln allele enhance the susceptibility to the development of CRC ( $\mathrm{p}=0.046, \mathrm{OR}=2.10)$. We also found a significant association between polymorphism and clinicopathological features, specifically in differentiated grade and lymph node metastasis. The Gln allele was detected more frequently in patients with well-differentiated grade and lymph node metastasis (N1) (OR=7.00 and 3.71, respectively). These data also suggest that this SNP may become a prognostic marker. Our findings suggest an association between the RAD18 Arg302Gln polymorphism and the risk of CRC. No other studies have found any association between human cancers and the RAD18 SNPs.

Although the molecular mechanism of the association between this SNP and cancer development is not yet clarified, several of the functions and functional domains of RAD18 were revealed. RAD18 is one of the most important proteins involved in the PRR pathway. While a number of proteins are known to be involved in the PRR pathway and RAD18 interacts with several of these proteins, the interaction with RAD6 in particular is essential for carrying out PRR $(5,6,10,11)$. RAD18 interacts with RAD6 through the RAD6 binding domain in the $\mathrm{C}$-terminal region (Fig. 2). RAD18 has several other functional domains, such as the RING-finger 
motif (25), zinc-finger motif (30,31) and E3 ubiquitin-ligase domain. The RING-finger motif, residing in the N-terminal region and the E3 ubiquitin-ligase domain together confer an ubiquitin-ligase activity on RAD18. The middle part of RAD18 contains a zinc-finger motif, which is considered to mediate protein-to-protein interaction or DNA binding. The RAD18 Arg302Gln polymorphism is located in the E3 ubiquitin-ligase domain (Fig. 2). Therefore, this SNP may affect the activity of RAD18, especially the ubiquitin-ligase activity. It is also possible that this SNP may affect the interaction between RAD18 and other proteins involved in PRR through its structural change, which is generated by the substitution of one amino acid residue, a basic amino acid residue (Arg), to a neutral residue (Gln). Considering that the Gln/Gln genotype was much more frequently found in the CRC patients than in the controls, the RAD18 with the Gln genotype may have decreased PRR activity and consequently may affect cancer development and progression.

In conclusion, our data provide evidence for an association between the RAD18 Arg302Gln polymorphism and the risk for the development and progression of CRC. It is possible that this polymorphism may influence susceptibility to a variety of human cancers through incomplete PRR. Further study with sufficiently larger populations and functional analysis of this polymorphism is needed in order to clarify this issue. Although the sample size we analyzed was small, the findings of this study are statistically significant. We expect that this study may contribute to the development of a novel strategy for the early diagnosis and prevention of CRC.

\section{Acknowledgements}

We thank the members of our University Hospital for their cooperation in specimen-sampling. This study was supported by a Grant-in-Aid for Scientific Research on Priority Area (No.12213084) to KS from the Ministry of Education, Culture, Sports, Science and Technology of Japan.

\section{References}

1. Hoeijmakers JH: Genome maintenance mechanisms for preventing cancer. Nature 411: 366-374, 2001.

2. Lawrence C: The RAD6 DNA repair pathway in Saccharomyces cerevisiae: what does it do, and how does it do it? Bioessays 16 : 253-258, 1994.

3. Sung P, Prakash S and Prakash L: Mutation of cysteine-88 in the Saccharomyces cerevisiae RAD6 protein abolishes its ubiquitinconjugating activity and its various biological functions. Proc Natl Acad Sci USA 87: 2695-2699, 1990.

4. Sung P, Prakash S and Prakash L: Stable ester conjugate between the Saccharomyces cerevisiae RAD6 protein and ubiquitin has no biological activity. J Mol Biol 221: 745-749, 1991.

5. Wood A, Schneider J, Dover J, Johnston M and Shilatifard A: The Paf 1 complex is essential for histone monoubiquitination by the Rad6-Bre1 complex, which signals for histone methylation by COMPASS and Dot1p. J Biol Chem 278: 34739-34742, 2003.

6. Bailly V, Lamb J, Sunq P, Prakash S and Prakash L: Specific complex formation between yeast RAD6 and RAD18 proteins: a potential mechanism for targeting RAD6 ubiquitin-conjugating activity to DNA damage sites. Genes Dev 8: 811-820, 1994.

7. Bailly V, Lauder S, Prakash S and Prakash L: Yeast DNA repair proteins Rad6 and Rad18 form a heterodimer that has ubiquitinconjugating, DNA binding and ATP hydrolytic activities. J Biol Chem 272: 23360-23365, 1997.

8. Bailly V, Prakash S and Prakash L: Domains required for dimerization of yeast Rad6 ubiquitin-conjugating enzyme and Rad18 DNA binding protein. Mol Cell Biol 17: 4536-4543, 1997.
9. Joazeiro CA and Weissman AM: RING-finger proteins: mediators of ubiquitin-ligase activity. Cell 102: 549-552, 2000.

10. Dohmen RJ, Madura K, Bartel B and Varshavsky A: The N-end rule is mediated by the UBC2 (RAD6) ubiquitin-conjugating enzyme. Proc Natl Acad Sci USA 88: 7351-7355, 1991.

11. Sung P, Berleth E, Pickart C, Prakash S and Prakash L: Yeast RAD6 encoded ubiquitin conjugating enzyme mediates protein degradation dependent on the N-end-recognizing E3 enzyme. EMBO J 10: 2187-2193, 1991.

12. Haracska L, Torres-Ramos CA, Johnson RE, Prakash S and Prakash L: Opposing effects of ubiquitin conjugation and SUMO modification of PCNA on replicational bypass of DNA lesions in Saccharomyces cerevisiae. Mol Cell Biol 24: 4267-4274, 2004.

13. Hoege C, Pfander B, Moldovan GL, Pyrowolakis G and Jentsch S: RAD6-dependent DNA repair is linked to modification of PCNA by ubiquitin and SUMO. Nature 419: 135-141, 2002.

14. Stelter P and Ulrich HD: Control of spontaneous and damageinduced mutagenesis by SUMO and ubiquitin conjugation. Nature 425: 188-191, 2003.

15. Kannouche PL, Wing $\mathbf{J}$ and Lehmann AR: Interaction of human DNA polymerase eta with monoubiquitinated PCNA: a possible mechanism for the polymerase switch in response to DNA damage. Mol Cell 14: 491-500, 2004.

16. Watanabe K, Tateishi S, Kawasuji M, Tsurimoto T, Inoue H and Yamaizumi M: Rad18 guides poleta to replication stalling sites through physical interaction and PCNA monoubiquitination. EMBO J 23: 3886-3896, 2004.

17. Tateishi S, Niwa H, Miyazaki J, Fujimoto S, Inoue $\mathrm{H}$ and Yamaizumi M: Enhanced genomic instability and defective post-replication repair in RAD18 knockout mouse embryonic stem cells. Mol Cell Biol 23: 474-481, 2003.

18. Yamashita YM, Okada T, Matsusaka T, et al: RAD18 and RAD54 cooperatively contribute to maintenance of genomic stability in vertebrate cells. EMBO J 21: 5558-5566, 2002.

19. Shekhar MP, Lyakhovich A, Visscher DW, Heng H and Kondrat N: Rad6 overexpression induces multinucleation, centrosome amplification, abnormal mitosis, aneuploidy and transformation. Cancer Res 62: 2115-2124, 2002.

20. Friedberg EC: DNA damage and repair. Nature 421: 436-440, 2003.

21. Ryk C, Kumar R, Thirumaran RK and Hou SM: Polymorphisms in the DNA repair genes XRCC1, APEX1, XRCC3 and NBS1 and the risk for lung cancer in never- and ever-smokers. Lung Cancer 54: 285-292, 2006

22. Ito H, Matsuo K, Hamajima N, et al: Gene-environment interactions between the smoking habit and polymorphisms in the DNA repair genes, APE1 Asp148Glu and XRCC1 Arg399Gln, in Japanese lung cancer risk. Carcinogenesis 25: 1395-1401, 2004.

23. Yamamoto $\mathrm{H}$, Hanafusa $\mathrm{H}$, Ouchida $\mathrm{M}$, et al: Single nucleotide polymorphisms in the EXO1 gene and the risk of colorectal cancer in a Japanese population. Carcinogenesis 26: 411-416, 2005.

24. Huang WY, Olshan AF, Schwartz SM, et al: Selected genetic polymorphisms in MGMT, XRCC1, XPD and XRCC3 and risk of head and neck cancer: a pooled analysis. Cancer Epidemiol Biomarkers Prev 14: 1747-1753, 2005

25. Costa S, Pinto D, Pereira D, et al: DNA repair polymorphisms may contribute differentially on familial and sporadic breast cancer susceptibility: a study on a Portuguese population. Breast Cancer Res Treat 103: 209-217, 2007.

26. Zhu Y, Lai M, Yang H et al: Genotypes, haplotypes and diplotypes of XPC and risk of bladder cancer. Carcinogenesis 28: 698-703, 2007.

27. Bolufer P, Barragan E, Collado M, Cervera J, López JA and Sanz MA: Influence of genetic polymorphisms on the risk of developing leukemia and on disease progression. Leuk Res 30: 1471-1491, 2006.

28. Hamajima N: PCR-CTPP: a new genotyping technique in the era of genetic epidemiology. Expert Rev Mol Diagn 1: 119-123, 2001.

29. Hamajima N, Saito T, Matsuo K, Kozaki K, Takahashi T and Tajima K: Polymerase chain reaction with confronting two-pair primers for polymorphism genotyping. Jpn J Cancer Res 91: $865-868,2000$.

30. Mackay JP and Crossley M: Zinc fingers are sticking together. Trends Biochem Sci 23: 1-4, 1998.

31. Akhtar A and Becker PB: The histone $\mathrm{H} 4$ acetyltransferase MOF uses a $\mathrm{C} 2 \mathrm{HC}$ zinc finger for substrate recognition. EMBO Rep 2: 113-118, 2001. 